\title{
Síndrome neuroléptico maligno y poliserositis en paciente usuaria de clozapina: una asociación infrecuente
}

\author{
Gonzalo Eymin $L^{1}$, Max Andresen $\mathbf{H}^{2}$, Jaime Godoy $\mathrm{F}^{3}$, \\ Gabriel Rada G 4 .
}

\section{Malignant neuroleptic syndrome and polyserositis associated to clozapine use. Report of one case}

Malignant neuroleptic syndrome is a complication of antipsychotic medication use. Clozapine use is also associated with polyserositis and eosinophilia. We report a 17 years old female treated with clozapine, valproic acid, lithium carbonate and lorazepam that consulted in the emergency room for confusion, lethargy, catatonia, rigidity, myalgya and fever. Complete blood count showed eosinophilia. An abdominal CAT scan showed ascites and pleural effusion. Clozapine was discontinued and bromocriptine was started. One week after admission, the patient remained febrile and liver enzymes were elevated. Valproic acid was discontinued. Inflammatory parameters stated to subside and the patient was discharged afebrile days after admission (Rev Méd Chile 2005; 133: 1225-8).

(Key Words: Bromocriptine; Clozapine; Neuroleptic malignant syndrome, neuroleptic-induced)

Recibido el 12 de abril, 2005. Aceptado el 23 de junio, 2005.

${ }^{1}$ Departamento de Medicina Interna. ${ }^{2}$ Departamento de Medicina Interna-Programa de Medicina Intensiva del Adulto. ${ }^{3}$ Departamento de Neurología. ${ }^{4}$ Residente de Medicina Interna, Hospital Clínico de la Pontificia Universidad Católica de Chile.

$\mathrm{E}^{1}$ síndrome neuroléptico maligno (SNM), es una reacción idiosincrásica conocida y temida del uso de antipsicóticos de primera generación. Sin embargo, se describe cada vez con mayor frecuencia SNM y cuadros símiles en relación al uso de nuevos antipsicóticos. En particular es conocida la asociación de la clozapina con este síndrome, describiéndose también en relación a

Correspondencia a: Gonzalo Eymin Lago. Marcoleta 367, Santiago, Chile. E-mail: eyminlago@yahoo.com ésta cuadros infrecuentes de poliserositis y eosinofilia. Sin embargo, la presencia simultánea de poliserositis y SNM no aparece descrita. A continuación se presenta el caso de una paciente presentó un SNM en concomitancia con poliserositis en relación al uso de clozapina.

\section{Caso CĹ́nico}

Paciente de 17 años, portadora de un trastomo bipolar, trastorno de personalidad limítrofe y con 
antecedente de abuso de marihuana, cocaína, éxtasis y heroína. Después de cuatro días de ser dada de alta desde una clínica psiquiátrica de Santiago, donde estuvo dos meses en tratamiento con clozapina, ácido valproico, carbonato de litio y lorazepam, inició un cuadro de confusión, letargia, conducta catatónica, rigidez, mialgias y fiebre, que mantenía por cinco días, razón por la que fue traída al Hospital Clínico de la Universidad Católica e ingresó a la Unidad de Cuidados Intensivos (UCI) con el diagnóstico presuntivo de meningoencefalitis. En el examen físico se constataron fiebre, taquicardia, normotensión, rigidez principalmente del esqueleto axial, y gran agitación psicomotora. El hemograma de ingreso mostró 17.000 leucocitos $/ \mathrm{mm}^{3}$ con $14 \%$ de baciliformes, plaquetas normales, VHS de 31, PCR de 19,8 mg/dl, CK de 920 $\mathrm{mg} / \mathrm{dl}$ con fracción $\mathrm{MB}$ de $22 \mathrm{mg} / \mathrm{dl}$. Se tomaron hemocultivos, cultivo de orina, radiografía de tórax y punción lumbar que fueron normales, pese a lo cual recibió antibióticos por sospecha de un cuadro pulmonar aspirativo. Dado que la paciente estaba recibiendo clozapina, se interpretó como un síndrome neuroléptico maligno, se suspendió la droga y se inició tratamiento con bromocriptina. Se le realizó un ecocardiograma de superficie que descartó endocarditis y una tomografía axial computada (TAC) de abdomen que reveló poliserositis, dada por ascitis y derrame pleural, que dada la escasa cuantía no se puncionaron. El estudio reumatológico mostró ANA, ANCA, anti-ADN, antiENA negativos y complementemia normal. A la semana del ingreso, la paciente se encontraba mejor de conciencia, pero persistía febril, agregándose marcada leucocitosis con desviación izquierda, eosinofilia mayor a $4.000 / \mathrm{mm}^{3}$, y alteración de pruebas hepáticas con SGOT de 627 U/L, LDH de $606 \mathrm{U} / \mathrm{L}$, fosfatasas alcalinas de $124 \mathrm{U} / \mathrm{L}$ y bilimubina de 1,1 mg/dL. En ese contexto, se suspendieron los antibióticos y el ácido valproico, quedando sólo con lorazepam. Permaneció en estas condiciones, con parámetros inflamatorios en descenso y 12 días después del ingreso cedió la fiebre. Al momento del alta estaba sin fiebre y con muy buen estado anímico, con anemia no regenerativa normocítica normocrómica, leucocitosis, sin desviación izquierda, eosinofilia mayor a $4.000 / \mathrm{mm}^{3}$, CK normal y pruebas hepáticas normales. En el control médico a los 10 días, la paciente se encontraba en buenas condiciones generales, deprimida, afebril, con CK normal y un hemograma con ascenso del hematocrito, sin eosinofilia, pero con linfocitosis relativa, alteraciones que se corrigieron en el hemograma de control después de 2 semanas.

\section{DisCUSIÓN}

El síndrome neuroléptico maligno es una complicación idiosincrática infrecuente del tratamiento con drogas antipsicóticas, siendo el haloperidol su mayor exponente. Se cree que su génesis radica en el bloqueo de receptores dopaminérgicos en el sistema nervioso central, o bien en el retiro de agonistas dopaminérgicos exógenos, como por ejemplo el retiro de levodopa en pacientes con enfermedad de Parkinson. La hipertermia resulta de una mayor actividad metabólica del miocito, así como de una alteración en la termorregulación hipotalámica. Los criterios diagnósticos se basan en la clínica, siendo las características fundamentales la rigidez muscular, hipertermia, alteración del estado mental e inestabilidad hemodinámica, asociada al uso de drogas antipsicóticas. Además de la hipertermia y rigidez debe haber leucocitosis y evidencias de laboratorio de injuria muscular, los síntomas deben mejorar al suspender la droga, y no debe aparecer nuevo déficit focal. Debe descartarse el uso de otras drogas que produzcan cuadros similares, como la metoclopramida, droperidol, halotano, etc.; el diagnóstico diferencial incluye catatonia letal, síndrome serotoninérgico, movimientos involuntarios secundarios al uso de antipsicóticos, el «heat stroke», status epiléptico, infección del sistema nerviosos central (SNC), trauma cerebral, neoplasias, porfiria aguda intermitente y tétano ${ }^{1}$. Las complicaciones incluyen infarto al miocardio, neumonía aspirativa, falla respiratoria, tromboembolismo, acidosis láctica y respiratoria, rabdomiolisis y falla renal.

La incidencia de SNM con antipsicóticos atípicos va de $0,02 \%$ a $2,44 \%$, siendo descrito en relación a clozapina, risperidona, olanzapina, quetiapina y amisulpirida. La mortalidad del síndrome en relación a estas drogas es menor que el producido por antipsicóticos clásicos ${ }^{2}$. Existen reportes de más de un episodio de SNM en un mismo paciente al utilizar 3 antipsicóticos diferentes ${ }^{3}$. A pesar de que la clozapina se ha usado con 
seguridad en pacientes que han sufrido un SNM por haloperidol, existen múltiples reportes en la literatura de SNM en relación a esta droga ${ }^{4-12}$. Por otra parte, hay evidencias que el uso de clozapina posterior al desarrollo de SNM por esta misma droga, no aumentaría el riesgo de volver a desarrollar el cuadro ${ }^{13,14}$. También hay reportes de SNM al usar conjuntamente clozapina con litio, o con carbamazepina ${ }^{15,16}$.

Karagianis et al revisaron en Medline todos los casos descritos entre 1966 y 1998, y encontró que las características clínicas más frecuentes del SNM por clozapina son taquicardia, confusión, y diaforesis $^{17}$. La fiebre, rigidez y aumento de CK son menos prominentes que en el SNM producido por antipsicóticos clásicos, e incluso está descrito el cuadro sin síntomas extrapiramidales ${ }^{18,19}$. En otra revisión, realizada por Tsai, se muestra que los factores asociados a un mayor riesgo de desarrollar SNM por clozapina son el antecedente de $\mathrm{SNM}$, presencia de lesión cerebral previa, uso concomitante de otro antipsicótico, ser hombre joven y tener una ferremia baja ${ }^{20}$. En cuanto al tratamiento, han demostrado ser útiles tanto el dantrolene como la bromocriptina ${ }^{20,21}$.

\section{REFERENCIAS}

1. GurRera RJ, Chang SS, Romero JA. A comparison of diagnostic criteria for neuroleptic Malignant Syndrome. J Clin Psychiatry 1992; 53: 56-62.

2. Ananth J, Parameswaran S, Gunatilake S, Burgoyne $\mathrm{K}$, SiDHom T. Neuroleptic Malignant Síndrome and atypical antipsychotic drugs. J Clin Psychiatry 2004; 65: 464-70.

3. Bottlender R, Jager M, Hofschuster E, Dobmeier $P$, MoLer HJ. Neuroleptic Malignant Syndrome due to atypical neuroleptics: three episodes in one patient. Pharmacopsychiatry 2002; 35: 119-21.

4. Baciewicz AM, Chandra R, Whelan P. Clozapineassociated Neuroleptic Malignant Syndrome. Ann Intern Med. 2002; 137 (5 part 1): 374.

5. Doan RJ., CaLaghan WD. Clozapine treatment and neuroleptic Malignant Syndrome. Can J Psychiatry 2000; 45: 394-5.

6. Trayer JS, Fidier DC. Neuroleptic Malignant Syndrome related to use of clozapine. J Am Osteopath Assoc. 1998; 98: 168-9.
Otra reacción adversa asociada al uso de clozapina es la poliserositis, cuya etiopatogenia no es clara, y que se manifiesta como derrame pericárdico hemorrágico, transudado o exudado pleural, acompañado de dolor torácico, fiebre, tos seca, calofríos, aumento de la VHS incluso sobre $100 \mathrm{~mm} / \mathrm{h}$ y de PCR sobre $10 \mathrm{~mm} / \mathrm{h}$. Existen varios reportes en la literatura de poliserositis aséptica con pruebas reumatológicas normales. En estos casos, la sintomatología se resuelve en 1 a 2 semanas de suspendida la clozapina, en tanto que las pruebas de laboratorio tardan hasta un mes en normalizarse $22-25$.

Este caso refleja que los antipsicóticos modernos no están ajenos a las complicaciones de los convencionales, y si bien el riesgo de desarrollarlas es menor, hay que tener un importante grado de sospecha frente a situaciones que se puedan presentar en pacientes bajo tratamiento con estas drogas, de modo de evitar confundirlas con trastornos conductuales propios de los problemas psiquiátricos de base, o bien con otros cuadros como procesos infecciosos del sistema nervioso central.

7. Dalkili A, Grosch WN. Neuroleptic Malignant Syndrome following initiation of clozapine therapy. Am J Psychiatry 1997; 154: 881-2.

8. Amore M, ZazZeri N, Berardi D. Atypical Neuroleptic Malignant Syndrome associated with clozapine treatment. Neuropsychobiology 1997; 35: 197-9.

9. THORNBERG SA, ERESHEFSKY L Neuroleptic Malignant Syndrome associated with clozapine monotherapy. Pharmacotherapy 1993; 13: 510-4.

10. DasGupta K, Young A. Clozapine-induced Neuroleptic Malignant Syndrome. J Clin Psychiatry 1992; 53: 294-5.

11. Muler DD, Sharafuddin MJ, Kathol RG. A case of clozapine-induced Neuroleptic Malignant Syndrome. J Clin Psychiatry 1991; 52: 99-101.

12. Stoudemire A, ClaYton L Succesful use of clozapine in a patient with a history of Neuroleptic Malignant Syndrome. J neuropsychiatry Clin Neurosci 1989; 1: 303-5.

13. Huang TL. Neuroleptic Malignant Syndrome associated with long-term clozapine treatment: report of a case and results of a clozapine rechallenge. Chang Gung Med J 2001; 24: 522-5. 
14. Chatterton R, Cardy S, Schramm TM. Neuroleptic Malignant Syndrome and clozapine monotherapy. Aust N Z J Psychiatry 1996; 30: 692-3.

15. Muler T, BecKer T, Fritze J. Neuroleptic Malignant Syndrome after clozapine plus carbamazepine. Lancet 1988; 2: 1500.

16. Pope HG, Cole JO, Choras PT, Fulwiler CE. Apparent neuroleptic malignant syndrome with clozapine and Lithium. J Nerv Ment Dis 1986; 174: 493-5.

17. Karagianis JL, Phimips LC, Hogan KP, LeDrew KK. Clozapine-associated neuroleptic malignant syndrome: two new cases and a review of the literature. Ann Pharmacother 1999; 33: 623-30.

18. Sachdev P, KruK J, KneEbone M, Kissane D. Clozapine-induced Neuroleptic Malignant Syndrome: review and report of new cases. J Clin Psychopharmacol 1997; 17: 233-4.

19. Goates MG, Escobar JI. An apparent neuroleptic malignant syndrome without extrapyramidal symptoms upon initiation of clozapine therapy: report of a case and results of a clozapine rechallenge. J Clin Psychopharmacol 1992; 12: 139-40.

20. Tsai G, Crisostomo G, Rosenblatt ML, Stern tA. Neuroleptic malignant syndrome associated with clozapine treatment. Ann Clin Psychiatry 1995; 7: 91-5.

21. Gankin L, Lichtenberg PS, Marcus EL, Munter RG. Suspected neuroleptic malignant syndrome in a patient receiving clozapine. Ann Pharmacother 1996; 30: 248-50.

22. Lim A, Sivakumaran P, Israel M. Clozapine-associated polyserositis. NZ Med J 2003; 116: U651.

23. Schönfeidt-Lecuona C, Conneman BJ. Sweet's syndrome and polyserositis with clozapine. Am J Psychiatry 2002; 159: 11.

24. Daly JM, Goldberg RJ, Braman SS. Polyserositis associated with clozapine treatment. Am J Psychiatry 1992; 149: 1274-5.

25. Catalano G, Catalano MC, Frankel Wetter RL. Clozapine induced polyserositis. Clin Neuropharmacol 1997; 20: 352-6. 